ИЗВЕСТИЯ АКАДЕМИИ НАУК ЭСТОНСКОП ССР. ФИЗИКА МАТЕМАТИКА PROCEEDINGS OF THE ACADEMY OF SCIENCES OF THE ESTONIAN SSR. PHYSICS * MATHEMATICS

$1985,34,1$

\title{
HIGH RESOLUTION NMR OF QUADRUPOLAR NUCLEI IN ROTATING POWDERS
}

\author{
(Presented by E. Lippmaa)
}

\section{Introduction}

Recent rapid advances of solid state NMR towards higher resolution have provided new possibilities for the elucidation of the structure of polycrystalline and amorphous solids, but practical applications have been largely confined to $\mathrm{I}=1 / 2$ nuclei, such as ${ }^{1} \mathrm{H},{ }^{13} \mathrm{C}$ and ${ }^{29} \mathrm{Si}$. Quite recently the magic angle sample spinning (MAS) technique has been extended to $\mathrm{I}=1$ nuclei, and interest in high resolution solid state NMR of quadrupolar nuclei with higher spins $(I>1)$ has likewise considerably grown during the past year.

In this letter we want to show the possibilities and limitations of fast sample spinning technique in high resolution NMR of quadrupolar nuclei in solids, and present a general formalism for calculating the NMR MAS spectra of quadrupolar nuclei with arbitrary spin. It appears that in the case of halfinteger spin, the derivation of chemical shift and parameters of quadrupolar interaction from the complicated experimental lineshape is rather simple. We have applied this technique with very good results to the study of $\mathrm{I}=3 / 2$ and $\mathrm{I}=5 / 2$ nuclei $\left({ }^{11} \mathrm{~B},{ }^{23} \mathrm{Na},{ }^{27} \mathrm{Al}\right)$.

\section{Theory}

In this section we concentrate our attention on the simplest case where evolution of the state of a single crystal spin system in an external magnetic field $B_{0}$ is governed by the one spin Hamiltonian containing only two terms

$$
\mathbf{H}=\mathbf{H}_{z}+\mathbf{H}_{Q},
$$

i.e. we disregard all other interactions except the Zeeman, isotropic chemical shift and quadrupole interaction. This is still the case often met in practice because in many samples homonuclear dipolar interactions between quadrupolar nuclei are rather weak due to dilution, heteronuclear dipolar couplings can be effectively removed by rf decoupling, and usually quadrupolar interaction is much larger than chemical shift anisotropy. Using conventional notation

$$
\begin{aligned}
& \mathbf{H}_{Z}=-\gamma(1-\sigma) B_{0} \mathbf{I}_{Z} \equiv \omega_{L} \mathbf{I}_{Z}, \\
& \mathbf{H}_{Q}=\frac{1}{3} \omega_{Q}\left\{V_{0}\left(\mathbf{I}^{2}-3 \mathbf{I}_{Z}^{2}\right)+\frac{1}{2} V_{-1}\left(\mathbf{I}_{Z} \mathbf{I}_{+}+\mathbf{I}_{+} \mathbf{I}_{Z}\right)+\right.
\end{aligned}
$$




$$
\left.+\frac{1}{2} V_{1}\left(\mathbf{I}_{Z} \mathbf{I}_{-}+\mathbf{I}_{-} \mathbf{I}_{Z}\right)+\frac{1}{2} V_{-2} \mathbf{I}_{+}^{2}+\frac{1}{2} V_{2} \mathbf{I}_{-}^{2}\right\} .
$$

Here the isotropic chemical shift $\sigma$ of the quadrupolar nucleus $\mathbf{I}$ is included in the Zeeman term, $\mathbf{H}_{z}$; and

$$
\omega_{Q}=\frac{3 e^{2} Q q}{4 I(2 I-1) \hbar} .
$$

Components of the electric field gradient (EFG) tensor, $V_{i}$ are functions of the asymmetry parameter $\eta$, of the Eulerian angles $\alpha, \beta, \gamma$ determining the orientation of PAS in the coordinate system fixed in the rotating sample with the $z$-axis (rotation axis) tilted by an angle $\theta$ from the polarizing magnetic field $B_{0}$, and there is a periodical time dependence with the rotation frequency $\omega_{r}$. One of the possible forms to write up the formulas of $V_{i}$ is the following:

$$
\begin{aligned}
& V_{0}=-\left(1-3 \cos ^{2} \theta\right) A+\frac{1}{2} \sin 2 \theta\left[(F-\mathrm{i} D) \mathrm{e}^{\mathrm{i}\left(\omega_{r} t-\alpha\right)}+\right. \\
&\left.+(F+\mathrm{i} D) \mathrm{e}^{-\mathrm{i}\left(\omega_{r} t-\alpha\right)}\right]+\frac{1}{2} \sin ^{2} \theta\left[(B-\mathrm{i} C) \mathrm{e}^{\mathrm{i} 2\left(\omega_{r} t-\alpha\right)}+\right. \\
&\left.+(B+\mathrm{i} C) \mathrm{e}^{-\mathrm{i} 2\left(\omega_{r} t-\alpha\right)}\right], \\
& V_{1}=V_{-1}^{*}=\mathrm{i} 3 A \sin 2 \theta+(\cos \theta-\cos 2 \theta)(D+\mathrm{i} F) \mathrm{e}^{\mathrm{i}\left(\omega_{r} t-\alpha\right)}+ \\
&+(\cos \theta+\cos 2 \theta)(D-\mathrm{i} F) \mathrm{e}^{-1\left(\omega_{r} t-\alpha\right)}+ \\
&+\sin \theta(1-\cos \theta)(C+\mathrm{i} B) \mathrm{e}^{\mathrm{i} 2\left(\omega_{r} t-\alpha\right)}+ \\
&+\sin \theta(1+\cos \theta)(C-\mathrm{i} B) \mathrm{e}^{-\mathrm{i} 2\left(\omega_{r} t-\alpha\right)}, \\
& V_{2}=V_{-2}^{*}=3 A \sin ^{2} \theta+\sin \theta(1-\cos \theta)(F-\mathrm{i} D) \mathrm{e}^{\mathrm{i}\left(\omega_{r} t-\alpha\right)}- \\
&-\sin \theta(1+\cos \theta)(F+\mathrm{i} D) \mathrm{e}^{-\mathrm{i}\left(\omega_{r} t-\alpha\right)}+ \\
&+\frac{1}{2}(1-\cos \theta)^{2}(B-\mathrm{i} C) \mathrm{e}^{\mathrm{i} 2\left(\omega_{r} t-\alpha\right)}+ \\
&+\frac{1}{2}(1+\cos \theta)^{2}(B+\mathrm{i} C) \mathrm{e}^{-\mathrm{i} 2\left(\omega_{r} t-\alpha\right)}
\end{aligned}
$$

where

$$
\begin{aligned}
& A=\frac{1}{4}\left[\left(1-3 \cos ^{2} \beta\right)-\eta \sin ^{2} \beta \cos 2 \gamma\right], \\
& B=\frac{1}{4}\left[3 \sin ^{2} \beta+\eta\left(1+\cos ^{2} \beta\right) \cos 2 \gamma\right] \\
& C=\frac{1}{2} \eta \cos \beta \sin 2 \gamma, \\
& D=\frac{1}{4} \sin 2 \beta(3-\eta \cos 2 \gamma), \\
& F=\frac{1}{2} \eta \sin \beta \sin 2 \gamma .
\end{aligned}
$$

For the following we demand that the Zeeman term predominates over the quadrupolar term

$$
\left|\omega_{L}\right| \gg\left|\omega_{Q}\right|
$$


which is in most cases guaranteed by the use of superconducting solenoids for the external magnetic field $B_{0}$.

The main assumption of our approach is that we take for granted that the relatively slow rotation-induced time dependence of the Hamiltonian (3) may be treated parametrically in the kinetic equation determining the evolution of the state of the spin system. This time dependence must be taken into account in the expressions for measurable quantities as a modulation.

This statement is based on the relative slowness of rotation relative to the Zeeman and quadrupole interactions - in practice the sample spinning frequency hardly exceeds $5 \mathrm{kHz}$, and at the same time the Larmor frequency $\omega_{L}$ is of the order of 10 to $100 \mathrm{MHz}$, and $\omega_{Q}$ is of the order of few $\mathrm{MHz}$.

With the use of the above-given assumption and of the condition (6), it is reasonable to use the interaction representation

$$
\tilde{\mathbf{H}}=\mathrm{e}^{\mathrm{i} \mathrm{H}_{\mathbf{z}} t} \mathrm{He}^{-\mathrm{i} \mathbf{H}_{\mathbf{z}} t},
$$

and to form for the problem an effective Hamiltonian over the Larmor period $T=2 \pi / \omega_{L}$ (and not over a rotation period $\omega_{r}^{-1}$ ). In the last step it is reasonable to confine oneself to the first approximation of the average Hamiltonian theory

$$
\mathbf{H}_{e f f}=\overline{\tilde{\tilde{H}}}_{Q}^{(0)}+\overline{\tilde{\mathbf{H}}}_{Q}^{(1)}
$$

where

$$
\begin{aligned}
& \overline{\mathbf{H}}_{Q}^{(0)}=\frac{1}{T} \int_{0}^{T} \tilde{\mathbf{H}}_{Q}(t) \mathrm{d} t, \\
& \tilde{\tilde{\mathbf{H}}}_{Q}^{(1)}=-\frac{\mathrm{i}}{2 T} \int_{0}^{T} \mathrm{~d} t^{\prime} \int_{0}^{t^{\prime}}\left[\tilde{\mathbf{H}}_{Q}\left(t^{\prime}\right), \tilde{\mathbf{H}}_{Q}\left(t^{\prime \prime}\right)\right] \mathrm{d} t^{\prime \prime},
\end{aligned}
$$

here the $V_{i}$ must be treated as constants.

Examination of the effective Hamiltonian with

$$
\begin{aligned}
& \overline{\dot{\tilde{H}}}(0)=\frac{1}{3} \omega_{Q} V_{0}\left(\mathbf{I}^{2}-3 \mathbf{I}_{Z}^{2}\right), \\
& \overline{\tilde{H}}_{Q}^{(1)}=-\frac{1}{18} k \omega_{Q}\left\{V_{1} V_{-1}\left(4 \mathbf{I}^{2}-8 \mathbf{I}_{z}^{2}-1\right) \mathbf{I}_{z}-V_{2} V_{-2}\left(2 \mathbf{I}^{2}-2 \mathbf{I}_{z}^{2}-1\right) \mathbf{I}_{z}+\right. \\
& +3\left[V_{0} V_{-1}\left(4 \mathbf{I}_{z}^{2}-4 \mathbf{I}_{z}+1\right) \mathbf{I}_{+}+V_{0} V_{1}\left(4 \mathbf{I}_{z}^{2}+4 \mathbf{I}_{z}+1\right) \mathbf{I}_{-}\right]+ \\
& \left.+6\left[V_{0} V_{-2}\left(\mathbf{I}_{Z}-1\right) \mathbf{I}_{+}^{2}+V_{0} V_{2}\left(\mathbf{I}_{Z}+1\right) \mathbf{I}_{-}^{2}\right]\right\} \text {, }
\end{aligned}
$$

allows us to establish that in a very good approximation, $\mathbf{H}_{\text {eff }}$ can be regarded as diagonal in the basis of $\mathbf{I}_{Z}$ eigenfunctions $|m\rangle$. The nondiagonal elements are either zero or smaller than the difference between the corresponding diagonal elements by at least the factor $k=\omega_{Q} / \omega_{L}$ throughout the whole rotation period $T_{r}$.

Consequently, the resonance frequency in the rotating frame of an arbitrary single quantum transition of the arbitrary spin I can be expressed as

$$
\omega_{m, m-1}=\left\langle m\left|\mathbf{H}_{e f f}\right| m\right\rangle-\left\langle m-1\left|\mathbf{H}_{e f f}\right| m-1\right\rangle .
$$


Of course, if the condition (6) is not well fulfilled, additional diagonalization of $\mathbf{H}_{e f f}$ is needed. In addition, in this case the use of higher orders of approximation in the effective Hamiltonian (8) is needed as well.

According to $(9)-(11)$

$$
\begin{aligned}
\omega_{m, m-1}= & -\omega_{Q} V_{0}(2 m-1)- \\
-\frac{1}{18} k \omega_{Q} & \left\{V_{1} V_{-1}[4 I(I+1)-24 m(m-1)-9]-\right. \\
& \left.\quad-V_{1} V_{-2}[2 I(I+1)-6 m(m-1)-3]\right\} .
\end{aligned}
$$

Remembering now the periodic time dependence of $V_{i}$ (period $T_{r}$ ) it is clear from (12) that every possible transition of every individual crystallite in the powder sample creates a central line at the mean value $\overline{\omega_{m, m-1}}$ of the frequency $\omega_{m, m-1}$ and numerous lines spaced by $\pm n \omega_{r}, n=1,2,3, \ldots$ from it as sidebands. The amplitudes of sidebands and, naturally, of the central lines depend upon the amplitudes of harmonics in (12), and are determined by the crystallite orientation and the parameters of quadrupolar and Zeeman interactions $\left(\omega_{Q}, \eta, \omega_{L}\right)$, the spinning angle $(\theta)$ and the observed transition $(\mathrm{m})$.

Relative to the formation of sidebands, notice a special feature of the central transition, $m=1 / 2$, of a halfinteger spin as compared with all other possible transitions, including the central transitions, $m=1,0$, of an integer spin. Namely, in this case the first term is absent in (12) and, consequently, the maximum values of harmonics are some orders of magnitude smaller than in other cases.

In the case of magic angle $\left(\cos ^{2} \theta=1 / 3\right)$ sample spinning

$$
\begin{aligned}
\overline{\omega_{m, m-1}}= & -\frac{1}{6} k \omega_{Q}\left\{\left(1+\frac{1}{3} \eta^{2}\right)[2 I(I+1)-14 m(m-1)-5]-\right. \\
& \left.-\frac{2}{9}\left(B^{2}+C^{2}+2 D^{2}+2 F^{2}\right)[6 I(I+1)-34 m(m-1)-13]\right\},
\end{aligned}
$$

the number of central lines in the spectrum equals $I$ when $I$ is an integer, and $\frac{1}{2}(2 I+1)$ otherwise. All the central lines, except those of the transition $\mathrm{m}=1 / 2$ of a halfinteger spin, are the sums of two components $\overline{\left(\omega_{m, m-1}\right.}=\overline{\left.\omega_{-m+1,-m}\right)}$.

If the deviation of the central line frequency (13) due to various crystallite orientations is smaller than $\omega_{r}$, then the powder pattern centerband and the spinning sidebands do not overlap. Assuming, in addition, random distribution of crystallites and supposing equality of the amplitudes of central lines of every crystallite, regardless of their orientation in powder sample, the powder pattern centerband lineshape can be easily calculated, since in this case it is determined by $\overline{\omega_{m, m-1}}$ only. As shown in Fig. 1, the resulting powder pattern lineshape is beautiful with clearly displayed singular points on it. Indeed, this lineshape features two peaks

$$
\omega_{p 1}= \begin{cases}-\frac{1}{8}\left(5-2 \eta+\frac{7}{3} \eta^{2}\right) a, & \text { for } 0 \leqslant \eta \leqslant \frac{3}{7} \\ -\frac{7}{4} a, & \text { for } \frac{3}{7} \leqslant \eta \leqslant 1\end{cases}
$$




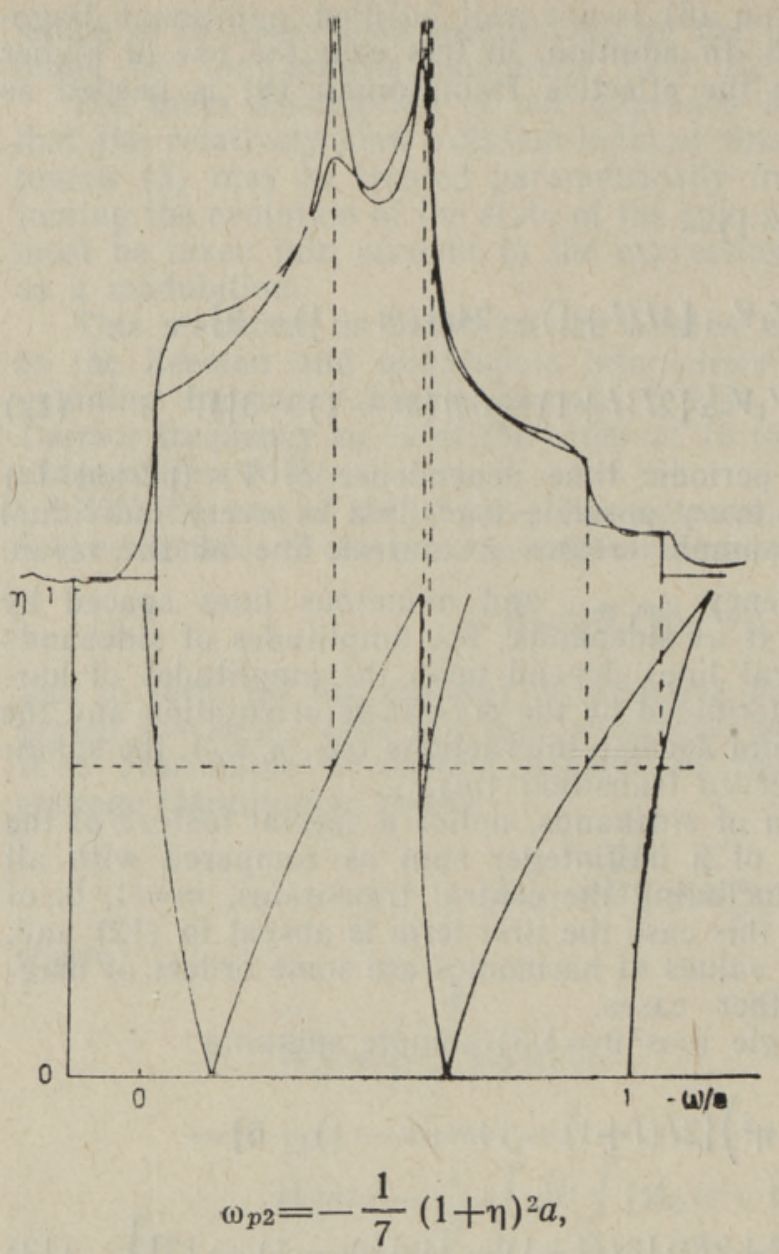

Fig. 1. The positions of singularities described by Eqs (13) and (14) as functions of the EFG tensor asymmetry parameter $\eta$ in the halfinteger spin central transition centerband lineshape. The experimental lineshape is that of ${ }^{23} \mathrm{Na} \quad(\mathbf{I}=$ $=3 / 2)$ in natrolite $\left(\omega_{Q} / 2 \pi=\right.$ $=1.76 \mathrm{MHz}, \eta=0.643)$. The Larmor frequency $\omega_{L}$ is taken as the zero point.

and one or two shoulders

$$
\begin{aligned}
& \omega_{s 1}=-\frac{1}{8}\left(5+2 \eta+\frac{7}{3} \eta^{2}\right) a, \\
& \omega_{s 2}=-\frac{1}{8}\left(5-2 \eta+\frac{7}{3} \eta^{2}\right) a, \quad \text { for } \frac{3}{7} \leqslant \eta \leqslant 1
\end{aligned}
$$

between the flanks at

$$
\begin{aligned}
& \omega_{\min }=-\left(1+\frac{1}{6} \eta^{2}\right) a \\
& \omega_{\max }=-\frac{1}{7}(1-\eta)^{2} a .
\end{aligned}
$$

Every crystallite in the sample gives the same contribution to the powder pattern lineshape in the case when the spinning frequency is much greater than all the amplitudes of the harmonic time dependence in (12). So the theoretical lineshape presented in Fig. 1 and the expressions (14) for the positions of singular points on it, are valid in the case of the central transition $m=1 / 2$ of a halfinteger spin where

$$
a=\frac{(2 I-1)(2 I+3)}{12} k \omega_{Q}
$$


Indeed, the experimental central lineshapes of halfinteger spins fit the theoretical ones well (see Figs. 1-3). Moreover, it follows from experiment that the singular points of this line do not move significantly with changes in the spinning frequency $\omega_{r}$ insofar as the central line and the spinning sidebands remain separate.

Let it be said that on the occasion of axial symmetry $\eta=0$ the halfinteger spin central transition centerband lineshape can be expressed analytically. The center of gravity of this line is shifted from the Larmor frequency $\omega_{L}$ by $\omega_{\epsilon}=-\frac{2}{5} a$.

The calculation of powder pattern central lineshapes of all other transitions, such as of the central transition of an integer spin $(m=1$, $m=0$ ), is more complicated because it requires the vastly different contributions of every individual crystallite to be taken into account. Since the contribution of a crystallite depends upon the parameters in the concrete experiment, it is rather difficult to give expressions for the lineshapes in these cases. Nevertheless, the positions of these powder pattern lineshapes are still determined by (13), which makes it possible to describe the full widths of these lines. For example, the integer spin central transitions centerband lineshape is placed, when $\eta=0$, between

$$
\omega_{\min }=-a \text { and } \omega_{\max }=-\left(1-\frac{2 b}{7}\right) a,
$$

where

$$
a=\frac{2 I(I+1)-5}{6} k \omega_{Q}, \quad b=\frac{6 I(I+1)-13}{2 I(I+1)-5} .
$$

It is something worth special notice that if one works with integer spins (or with others than the central transition of a halfinteger spin) then in order to register the central lineshape with as high a resolution as possible (such as predicted by (13)), an extremely precise and stable magic angle setting of the spinner is necessary. Due to the absence of the first term in (12), the halfinteger spin central transition lineshape, firstly, is less sensitive to the accuracy of the angle setting, and secondly, the maximum narrowing of it occurs not at the magic angle of the spinner axis. In the case of $\eta=0$, it is the angle of $\theta=\arccos \sqrt{\frac{15-2 \sqrt{30}}{35}}$ $\left(\theta=70.124^{\circ}\right)$ when the powder pattern is located in the frequency interval of

$$
-\frac{9}{14} \frac{13+2 \sqrt{30}}{35} a \text { to }-\frac{9}{14} \frac{23-4 \sqrt{30}}{35} a .
$$

Of course, at the non-magic angles the interactions neglected in this analysis (for example, the chemical shift anisotropy) are more significant.

In conclusion let it be said that if there exist, in addition to the quadrupolar spin $I$, a $S=1 / 2$ spin in the single crystal spin system with dipolar interaction between them, then the halfinteger $I$ central transition centerband lineshape is shifted from the position predicted by (14) by an amount proportional to $\frac{\omega_{D}}{\omega_{L}} \omega_{D}$; where $\omega_{D}=\frac{3}{2} \frac{\gamma_{I} \gamma_{S} \hbar^{2}}{r^{3}}$ and it is somewhat distorted. All the other powder pattern lineshapes of quadrupolar spins are influenced also by the loss of coincidence of the lines from single crystal symmetrical transitions $\left.\overline{\left(\omega_{m, m-1}\right.} \neq \overline{\omega-m+1,-m}\right)$.

At the same time the dipolar interaction with a quadrupolar nucleus 


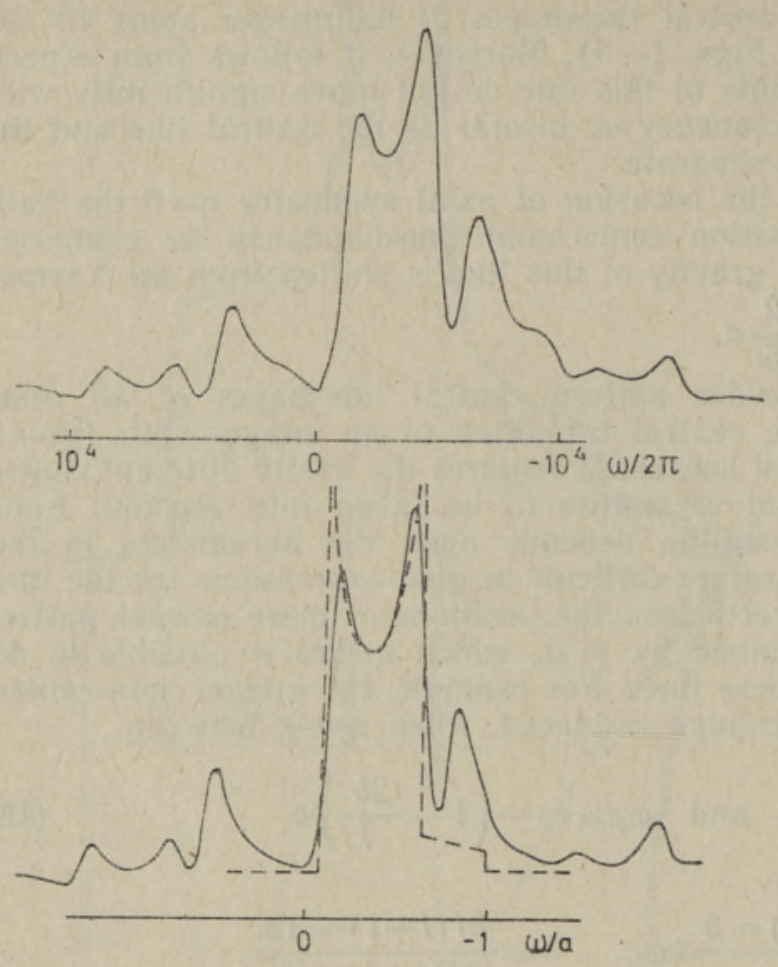

Fig. 2. Numerically calculated (lower) and experimental (upper) MAS-NMR central transition spectra of ${ }^{11} \mathrm{~B} \quad(\mathrm{I}=$ $=3 / 2$ ) in solid $\mathrm{B}_{2} \mathrm{O}_{3}, \omega_{Q} / 2 \pi=$ $=2.76 \mathrm{MHz}, \eta=0$. Dashed line represents the theoretical powder pattern lineshape determined by formula (13), solid line the same when first two sidebands are included, assuming Gaussian line broadening.

appears in the $S$ spin MAS spectra via splitting of the $S$ resonance into a multiplet and broadening of its components. The maximum number of components equals $I+1$, if $I$ is an integer, and $\frac{2 I+1}{2}$ if $I$ is a halfinteger spin. All the multiplet components, except that of corresponding to $m=0$ of an integer $I$, are the sums of two lines.

\section{Experiment}

The theory presented above was tested in experiments on quadrupolar nuclei with halfinteger spins $I=3 / 2,5 / 2$, such as ${ }^{11} \mathrm{~B},{ }^{23} \mathrm{Na}$ and ${ }^{27} \mathrm{Al}$. The lineshape measurements were performed on a Bruker CXP-200 NMR spectrometer in a $47 \mathrm{kGauss}$ field. The EFG tensor anisotropy $\eta$ varies in the investigated media in the interval between 0 and 0.94 , and the intensity of quadrupolar interaction between $0.334 \mathrm{MHz}$ and $2.95 \mathrm{MHz}$. Proton decoupling was used in some cases and MAS rotation frequency was varied between 3 and $5 \mathrm{kHz}$, which enables to separate the centerband spectral features from the spinning sidebands. Good agreement between the experimental and calculated lineshapes of the central transitions was found in all cases.

In Figures 2 and 3 two theoretical curves are presented, one of which corresponds to Eq. 13 and the second takes into account either the sideband formation from the central transition (Fig. 2, only first and second sidebands were included), or some Gaussian broadening of the lines from every crystallite in the powder (Fig. 3).

The observation of non-central transitions of a halfinteger spin separately from the central transition is difficult due to the greatly different intensities. Nevertheless, it was possible to measure the frequency shift between the central and coincident $\pm 3 / 2 \leftrightarrow \pm 1 / 2$ transitions of $a$ 

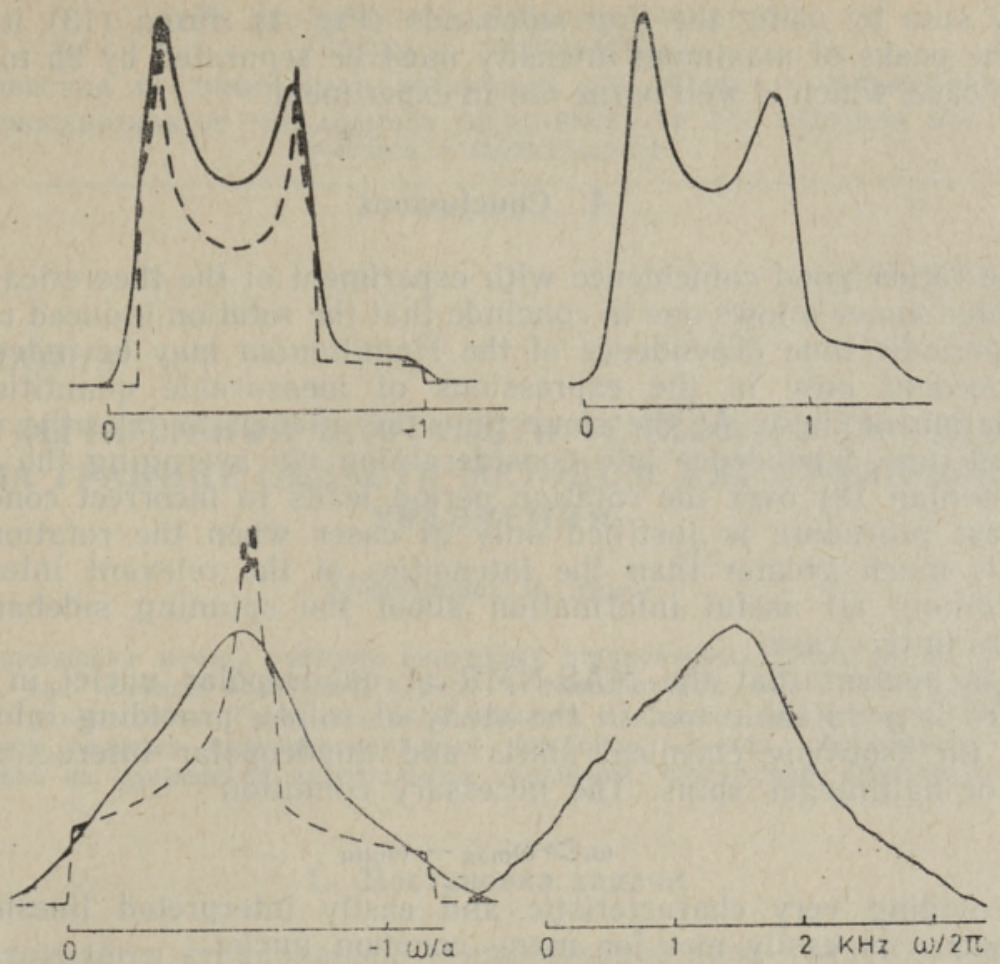

Fig. 3. Numerically calculated (left) and experimental (right) central transition lineshapes of halfinteger spins. Dashed lines - calculated from Eq. (13), solid lines - with additional Gaussian broadening. Upper row: ${ }^{23} \mathrm{Na}(I=3 / 2)$ in sodium nitrite $\mathrm{NaNO}_{2}-$ $-\omega_{\nu} / 2 \pi=1.1 \mathrm{MHz}, \eta=0.109$. Lower row: ${ }^{27} \mathrm{Al} \quad(\mathbf{I}=5 / 2)$ in spodumene $-\omega_{0} / 2 \pi=$ $=2.95 \mathrm{MHz}, \eta=0.94$.

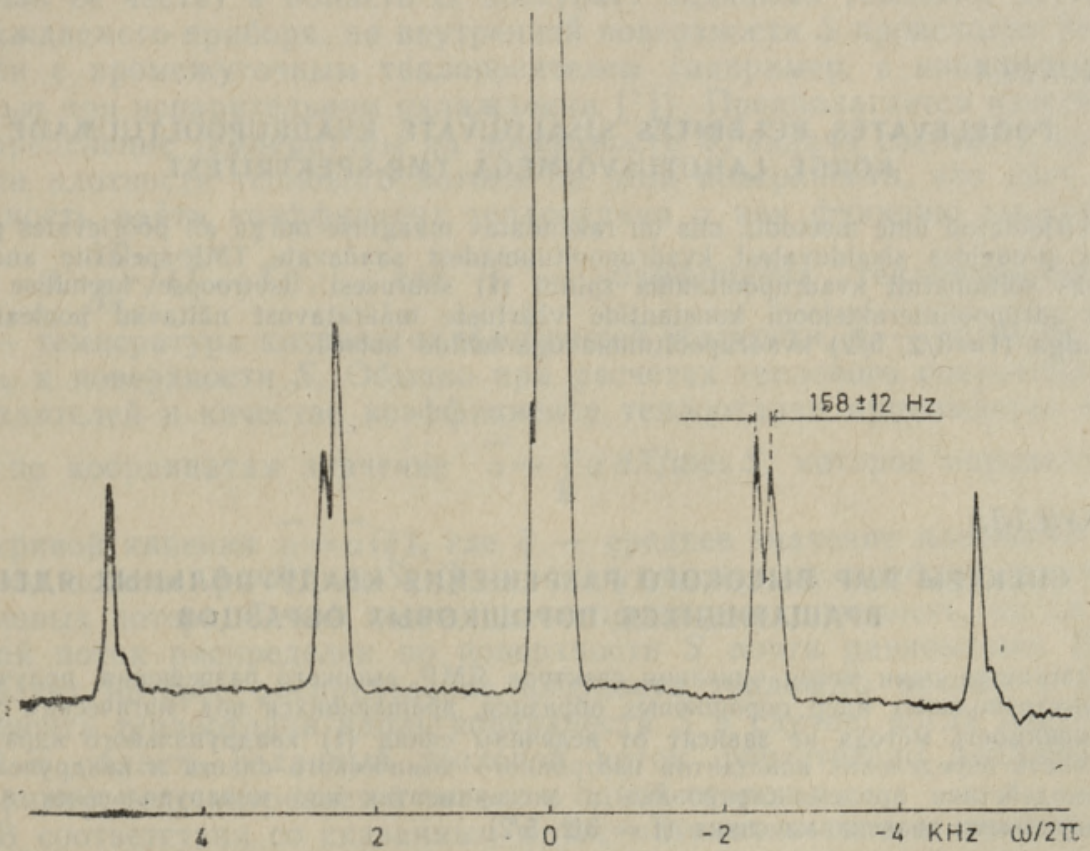

Fig. 4. Experimental NMR-MAS spectra of ${ }^{23} \mathrm{Na}(I=3 / 2)$ in sodium nitrate $\mathrm{NaNO}_{3}-$ $-\omega \circ / 2 \pi=0.334 \mathrm{MHz}, \eta=0$. The spinning sidebands enable to determine the frequency shift between the non-central $( \pm 3 / 2 \leftrightarrow \pm 1 / 2)$ and central $(1 / 2 \leftrightarrow-1 / 2)$ transitions. 
$I=3 / 2$ spin by using the first sidebands (Fig. 4). From (13) it follows that the peaks of maximum intensity must be separated by 95 to $220 \mathrm{~Hz}$ in this case, which is well borne out in experiment.

\section{Conclusions}

The rather good coincidence with experiment of the theoretical results presented above allows one to conclude that the rotation-induced relatively slow periodic time dependence of the Hamiltonian may be indeed taken into account only in the expressions of measurable quantities as a modulation of these. At the same time the attempt to take the rotationinduced time dependence into considerataion via averaging the effective Hamiltonian (8) over the rotation period leads to incorrect conclusions. The last procedure is justified only in cases when the rotation frequency is much greater than the intensities of the relevant interactions. In addition, all useful information about the spinning sidebands disappears in this case.

It is evident that the MAS-NMR of quadrupolar nuclei in powder samples is a valuable tool in the study of solids, providing information about the isotropic chemical shifts and quadrupolar interaction constants of halfinteger spins. The necessary condition

$$
\omega_{r}>\omega_{\max }-\omega_{\min }
$$

for providing very characteristic and easily interpreted lineshapes in the spectra is easily met for many common nuclei.

Academy of Sciences of the Estonian SSR,

Received

Institute of Chemical Physics and Biophysics

\section{E. KUNDLA}

\section{PÖORLEVATES PULBRITES SISALDUVATE KVADRUPOOLTUUMADE KORGE LAHUTUSVOIMEGA TMR-SPEKTRITEST}

On kirjeldatud ühte meetodit, mis on rakendatav maagilise nurga all pöörlevates pulbrilistes proovides sisalduvatest kvadrupooltuumadest saadavate TMR-spektrite analüüsimiseks sõltumatult kvadrupooltuuma spinni (I) suurusest. Isotroopse keemilise nihke ja kvadrupoolinteraktsiooni konstantide väärtuste määratavust näitavad poolearvulise spinniga $(\mathbf{I}=3 / 2,5 / 2)$ kvadrupooltuumadega tehtud katsed.

\section{Э. КУНДЛА}

\section{СПЕКТРЫ ЯМР ВЫСОКОГО РАЗРЕШЕНИЯ КВАДРУПОЛЬНЫХ ЯДЕР ВРАЩАЮЩИХСЯ ПОРОШКОВЫХ ОБРАЗЦОВ}

Предлагается один метод описания спектров ЯМР высокого разрешения, полученных от квадрупольных ядер порошковых образцов, вращающихся под магическим углом. Применимость метода не зависит от величины спина (I) квадрупольного ядра. Возможность определения константов изотропного химнческого сдвига и квадрупольного взаимодействия продемонстрирована в экспериментах над квадрупольными ядрами с полуцелыми значениями спина $(I=3 / 2,5 / 2)$. 